Forthcoming in: SYNTHESE

\title{
Ramsification and Inductive Inference
}

\section{Panu Raatikainen}

\begin{abstract}
An argument, different from the Newman objection, against the view that the cognitive content of a theory is exhausted by its Ramsey sentence is reviewed. The crux of the argument is that Ramsification may ruin inductive systematization between theory and observation. The argument also has some implications concerning the issue of underdetermination.
\end{abstract}

\section{Introduction}

Scientific realism proposes that we are justified in believing that successful theories of mature science are at least approximately true, and that the unobservable theoretical entities they postulate really exist. The standard argument in favour of this view is the so-called "nomiracles argument": the practical and observational success of science would be miraculous if scientific theories were not at least approximately true descriptions of the world, and the theoretical objects they postulate did not exist. Then again, arguments exist which seem to undermine scientific realism, and the no-miracles argument. Most importantly, perhaps, there are arguments which lean on the actual history of science and radical theory changes therein for example, the notorious "pessimistic meta-induction", and also various less general arguments to the same effect. Roughly, the thesis is that many past theories in science have turned out to be to a large extent false, and their theoretical terms non-referring; therefore - it is concluded - it is not justified to expect that the theoretical entities postulated by present theories exist either (see Laudan 1981).

The view known as Structural Realism has emerged over the past two decades and seems to enjoy some popularity among philosophers of science. It aims to be a middle-of-the-road view between full-blown scientific realism and empiricist antirealism which is thoroughly sceptical about unobservable theoretical entities (and theories dealing with them), and to take both these oppositional arguments seriously. Very roughly put, the epistemological form of structural realism holds that our knowledge of the world is limited to its structure; the metaphysical form of structural realism holds that in fact it is only structure that exists independently. 
However, the arguments from the history of science are not the only type of arguments which threaten scientific realism. In particular, any sort of realism, whether structural or of a more standard kind, must be able the reply to the challenge of arguments from the alleged radical underdetermination of theory by observation. The standard response is to grant that deductive underdetermination (i.e. that the theory cannot be logically derived from the evidence; or, that two incompatible theories may have exactly the same deductive observational consequences) is unavoidable, but insist that we also need to take into account the inductive relationships between observations and theory - and that this allows one to discard any radical underdetermination (see e.g. Laudan 1990, 1998; Ladyman 2002; Psillos 2005). Anyone willing to defend even a modest rationality of science must therefore allow considerations concerning inductive and not only deductive relations between theories and observations. And this has some relevance in what follows.

In contemporary philosophy, structural realism was introduced by John Worrall (1989), though it arguably has some prominent predecessors. He considered in particular the development of the theory of light in physics and the transition from ether theory to the theory of the electromagnetic field - one of the most difficult historical cases for scientific realism to accommodate. Worrall suggests that there was, after all, an important element of continuity in this shift; that more than just empirical content was preserved in it - namely, the form or structure of the former theory. However, Worrall adds, the content of the theories is radically different. More generally, he submitted that we should not believe that our scientific theories can manage to discover the nature of the postulated theoretical entities, as standard scientific realism suggests; rather, we should only commit ourselves to the structural content of our theories. Yet, this is more than only their empirical content, which is all that empiricist antirealism allows. It is proposed that such structural realism can both avoid the difficulties pessimistic meta-induction etc. raises and does not make the success of science miraculous it is "the best of both worlds". Structural realism has found many advocates in the philosophy of science. (See Ladyman 2008 for an excellent overview.)

The concept of 'structural content' of a scientific theory, essential for structural realism, obviously needs clarification. One standard way of doing this, advocated by Worrall himself as well as by Elie Zahar, for example, is in terms of Ramsey sentences. Worrall and Zahar (2001), in particular, argue that the cognitive content of a theory is exhausted by its Ramsey sentence. There are now also other ways of working out the idea of structural content (see 
Ladyman 2008), but the variant that utilizes Ramsification is certainly popular. I shall call the brand of structural realism which explicates structural content in this way "Ramsifying Structural Realism”. In what follows, I focus solely on this sort of structural realism.

More generally, the view that the factual content of a theory is faithfully captured by its Ramsey sentence seems to be quite popular in philosophy. For example, David Lewis, Frank Jackson, and a number of philosophers following them in the so-called Canberra Plan (see e.g. Braddon-Mitchell \& Nola 2009) make a heavy use of Ramsey sentences, and apparently hold related views. The arguments below are directed against all such views which hold the cognitive content of a theory is exhausted by its Ramsey sentence.

\subsection{Theories and Their Ramsey Sentences}

If the theory $S$ is presented in a standard form with theoretical predicates and relations $T_{1}, T_{2}$ $\ldots, T_{\mathrm{n}}$, and observational predicates and relations $O_{1}, O_{2} \ldots, O_{\mathrm{m}}$, the Ramsey sentence $S^{\mathrm{R}}$ of $S$ is obtained by first replacing all the theoretical predicates with second-order variables, and then, to the result of this replacement, prefixing the existential quantifiers with respect to those second order variables. Thus, if the original theory $S$ is written as

$$
S\left(T_{1}, T_{2} \ldots, T_{\mathrm{n}}, O_{1}, O_{2} \ldots, O_{\mathrm{m}}\right),
$$

then $S^{\mathrm{R}}$ is:

$$
\left(\exists X_{1}\right)\left(\exists X_{2}\right) \ldots\left(\exists X_{\mathrm{n}}\right) S\left(X_{1}, X_{2} \ldots, X_{\mathrm{n}}, O_{1}, O_{2} \ldots, O_{\mathrm{m}}\right)
$$

Here are some important logical properties of Ramsey sentences (cf. Psillos 2006) (the background logic assumed is a suitable system of second-order logic):

$S^{\mathrm{R}}$ is a logical consequence of $S$.

$S^{\mathrm{R}}$ has exactly the same first-order observational consequences as $S$.

$S_{1}$ and $S_{2}$ have incompatible observational consequences iff $S_{1}{ }^{\mathrm{R}}$ and $S_{2}{ }^{\mathrm{R}}$ are incompatible. 
$S_{1}$ and $S_{2}$ may make incompatible theoretical assertions and yet $S_{1}{ }^{\mathrm{R}}$ and $S_{2}{ }^{\mathrm{R}}$ can be compatible.

If $S_{1}{ }^{\mathrm{R}}$ and $S_{2}{ }^{\mathrm{R}}$ are compatible with the same observational truths, then they are compatible with each other.

Thus, at first sight, Ramsey sentences seem to suit well the purposes of structural realism, and to capture the cognitive contents of theories.

\subsection{The Newman Objection}

There is, however, a popular objection to Ramsifying Structural Realism and related views, the idea of which goes back to Newman's (1928) critique of Russell's version of structuralism - hence its name, the "Newman objection". ${ }^{1}$ The objection was revitalized in contemporary debate by Demopoulos and Friedman (1985). Generally, the objection is that if a theory is just consistent, and observationally adequate, ${ }^{2}$ then the corresponding Ramsey sentence is almost trivially true, provided that the domain only has a sufficient cardinality. Therefore, the objection continues, Ramsifying Structural Realism collapses into a radical empiricist denial of theoretical entities, and cannot provide a middle ground between that view and full-blown realism with respect to these entities. (For some complications, and for a careful and rigorous development of the objection, see Ketland 2004). Cruse (2005) and Melia and Saatsi (2006), on the other hand, subtly defend the Ramsey sentence approach against the Newman objection. It may well be that the Newman objection can be further defended against these critiques (cf. Ketland 2009, Ainsworth 2009). But at the moment, there does not seem to be a wide consensus concerning the issue, and the fate of the Newman objection remains somewhat open.

\section{Argument from Inductive Systematization}

Be that is it may, there is another line of reasoning, independent of the Newman objection, which poses a serious problem for any view which contends that the cognitive content of a 
theory is exhausted by its Ramsey sentence - an argument of which all parties of the present debate seem to be ignorant. It concerns the inductive rather than deductive relations between observations, theories and their Ramsey sentences. And we have seen above that because of the threat of radical underdetermination, it is in any case obligatory to take into account such inductive relations as well.

The idea was briefly suggested by Hempel (1958), developed in much more detail by Scheffler (1963, 1968), and culminated in Niiniluoto's conclusive though unfortunately little known defence of this argumentation strategy (Niiniluoto 1972, 1973; see also Tuomela 1973, 1974). As philosophers today seem to be almost universally ignorant of these arguments, it is perhaps proper to remind the philosophical community about them and review the main points.

\subsection{Inductive Systematization}

The principal conclusion of the argument is that Ramsification ruins inductive systematization between theories and observation. The notion of inductive systematization was introduced by Hempel (1958). It was discussed in the philosophy of science in the 1960s and early 70s (see Niiniluoto 1972), but seems to have disappeared from the philosophy of science literature. Yet it is a quite natural and relevant general concept.

By the term "systematization", Hempel simply meant any deductive or inductive argument which has as its premises both some singular statements and one or more laws, and as its conclusion a singular statement (or empirical law), and which serves as an explanation, prediction or postdiction (inferring the past), or something similar.

It is illuminating to consider first the notion of deductive systematization: Let $S$ be a theory formulated in a language $L$, and let $L_{O}$ be the observational sublanguage of $L$. Let $e$ and $h$ be some contingent statements of $L_{O}$.

A theory $S$ achieves deductive systematization (with respect to $L_{O}$ ) iff

$h$ is deducible from $S \wedge e$, $h$ is not deducible from $e$, 
for some factual observational $e$ and $h$.

Let $I$ then be an inducibility relation characterized by some set of inductive rules. The following arguments are relatively independent of the particular choice of rules.

We may note in passing, though, that two standard explications for inducibility are as follows (where $p$ is some probability measure defined for the language):

(i) Positive relevance criterion

$$
e \text { induces } h \text { iff } p(h / e)>p(h) \text {. }
$$

(ii) High probability criterion

$$
e \text { induces } h \text { iff } p(h / e)>k \text {, for some constant } k>0.5 \text {. }
$$

Be that as it may, the notion of inductive systematization is then completely analogous to that of deductive systematization, except that one adds the condition that the theory and established empirical evidence do not even deductively entail the induced statement at stake.

$\mathrm{S}$ is said to achieve inductive systematization (with respect to $L_{O}$ ) if and only if

(i) $I(S \wedge e, h) \quad h$ is inducible from $(S \wedge e)$

(ii) $\neg I(e, h) \quad \mathrm{h}$ is not inducible from $e$ alone

(iii) $h$ is not deductively derivable from $S \wedge e$.

\subsection{Scheffler's Argument}

Following Hempel's (1958) suggestion, Scheffler (1963, 1968) argued that Ramsification may fail to preserve inductive systematization. Here is Scheffler's argument:

Consider the following simple theory $S_{1}$, where $O_{1}$ and $O_{2}$ are assumed to be (possibly complex) observational predicates, and $T$ a theoretical predicate:

$S_{1}$

$$
(\forall x)\left[\left(T(x) \rightarrow O_{1}(x)\right) \wedge\left(T(x) \rightarrow O_{2}(x)\right)\right]^{3}
$$


By Ramsifying this, we get

$S_{1}{ }^{\mathrm{R}} \quad(\exists X)(\forall x)\left[\left(X(x) \rightarrow O_{1}(x)\right) \wedge\left(X(x) \rightarrow O_{2}(x)\right)\right]$

Now $S_{1}$ is non-analytic, but $S_{1}{ }^{\mathrm{R}}$, its Ramsey sentence, is in fact a truth of second-order logic and apparently analytic. That is, in this simple case, Ramsification converts a substantive first-order theory into a second-order logical truth and thus entails a loss of substantive content. Exactly the same difference occurs with the theory

$S_{2} \quad(\forall x)\left[\left(T(x) \rightarrow O_{1}(x)\right) \wedge\left(T(x) \rightarrow \neg O_{2}(x)\right)\right]$,

and its Ramsification:

$S_{2}{ }^{\mathrm{R}} \quad(\exists X)(\forall x)\left[\left(X(x) \rightarrow O_{1}(x)\right) \wedge\left(X(x) \rightarrow \neg O_{2}(x)\right)\right]$

Note now that as $S_{1}{ }^{\mathrm{R}}$ and $S_{2}{ }^{\mathrm{R}}$ are both logical truths of second-order logic, they are logically equivalent. Scheffler next submits that $S_{1}$ achieves inductive systematization between $O_{1}(a)$ and $O_{2}(a){ }^{4}$ However, if $S_{1}{ }^{\mathrm{R}}$ also achieved inductive systematization between $O_{1}(a)$ and $O_{2}(a)$, it would follow that, analogously, $S_{2}{ }^{\mathrm{R}}$ would achieve inductive systematization between $O_{1}(a)$ and $\neg O_{2}(a)$. However, $S_{2}{ }^{\mathrm{R}}$ is logically equivalent to $S_{1}{ }^{\mathrm{R}}$. Consequently, they cannot possibly achieve inductive systematization between both $O_{1}(a)$ and $O_{2}(a)$, and $O_{1}(a)$ and $\neg O_{2}(a)$. Therefore, Scheffler concludes, $S_{1}{ }^{\mathrm{R}}$ cannot achieve inductive systematization between $O_{1}(a)$ and $\mathrm{O}_{2}(a)$, and thus Ramsification of a theory can ruin inductive systematization.

So how does the original theory $S_{1}$ achieve inductive systematization between $O_{1}(a)$ and $\mathrm{O}_{2}(a)$ ? One might reason as follows (but see below). Assume that there is an individual $a$ which shows (according to $S_{1}$ ) the symptoms of $T$, i.e., $O_{1}(a)$. This evidence is supposed to give inductive support for $T(a)$, by the first conjunct of $S_{1}$. One can then, by the second conjunct of $S_{1}$, conclude $O_{2}(a)$. Thus, so the reasoning goes, $S_{1}$ achieves inductive systematization between $O_{1}(a)$ and $O_{2}(a)$. 


\subsection{Critical Responses to Scheffler's argument}

Scheffler's argument, however, has not remained unchallenged. Convincing as it may at first appear, two objections have been raised against it.

First, under closer scrutiny, it turns out that the reasoning (see above) to the effect that $S_{1}$ achieves inductive systematization between $O_{1}(a)$ and $O_{2}(a)$ uses two principles which cannot both hold for inducibility:

"The rule of converse entailment": If $h$ is deducible from $e$, then $e$ is inducible from $h$ - used when inducing $T(a)$ from $O_{1}(a)$;

"The rule of special consequence": If $h$ is inducible from $e$ and $b$ is deducible from $h$, then $b$ is inducible from $e$ - used when going from $T(a)$ to $O_{2}(a)$.

However, it is well known that these rules of induction are not acceptable together: jointly they entail that every statement confirms every other ${ }^{5}$ (Hempel 1945; Skyrms 1966; Hesse 1970a, 1970b; Niiniluoto 1972). Hence, Scheffler's argument - as it stands - fails. ${ }^{6}$

Second, it has been argued that even the original theory $S_{1}$ "has no empirical content" (Hooker 1968), is "immune to observational confirmation" (Bohnert 1968) or is "empirically trivial" (Stegmüller 1970), and therefore cannot possibly achieve inductive systematization in the first place.

\subsection{The Objections Answered}

As Niiniluoto (1972) points out, the basic difficulty in the accounts of Scheffler, Hempel and others is that they attempt to show that inductive systematization is established indirectly, that is, by means of at least two-step arguments going 'through' theories, as it were, via theoretical terms; for example, that $O_{2}(a)$ may be induced from $O_{1}(a)$ assuming $S_{1}$ in two steps, first from $O_{1}(a)$ to $T(a)$, and then from $T(a)$ to $O_{2}(a)$. This idea presupposes transitivity conditions that no reasonable explicate of the inducibility relation seems to possess. 
Niiniluoto (1972), however, shows that this problem can been solved by interpreting the condition (i) of the definition of inductive systematization as a one-step inductive argument. No transitivity problems then arise. That is, one may interpret the condition " $h$ is inducible from $e$ and $T$ ', as a one-step inductive argument from the conjunction ' $T \wedge e$ ' to ' $h$ ' - that is, in Scheffler's case, from the conjunction

$$
O_{1}(a) \wedge(\forall x)\left[\left(T(x) \rightarrow O_{1}(x)\right) \wedge\left(T(x) \rightarrow O_{2}(x)\right)\right]
$$

to $\mathrm{O}_{2}(a)$.

According to this suggestion, theories play a direct, rather than indirect or mediating, role in the establishment of inductive systematization. Interpreting the case in this way, Scheffler's argument can be saved from the first objection.

Put differently, Niiniluoto (1972) makes evident that Scheffler's argument is valid given the following principle:

$$
\begin{aligned}
& \text { If } S \text { achieves inductive systematization between } e \text { and } h \text {, and } S^{\prime} \text { is logically } \\
& \text { equivalent to } S \text {, then } S^{\prime} \text { does not achieve inductive systematization between } \\
& e \text { and } \neg h \text {. }
\end{aligned}
$$

And this principle is satisfied by all the most natural explicates of inducibility, for example the positive relevance criterion and the high probability criterion (see above).

But what about the influential objection to Scheffler's argument (by Hooker, Bohnert, and Stegmüller), according to which even the original, non-ramsified theory $S_{1}$ does not achieve inductive systematization, because it has no deductive observational content? Consider the following three statements:

$\left(1^{*}\right)$ Theory $S$ has no contingent deductive observational consequences.

$(2 *)$ Theory $S$ is not observationally testable.

$\left(3^{*}\right)$ Theory $S$ does not establish inductive systematization (w.r.t. $L_{O}$ ). 
The objection of Bohnert, Hooker and Stegmüller to Scheffler's argument supposes the following:

$$
(1 *) \Rightarrow(2 *)
$$

$$
\left(2^{*}\right) \Rightarrow\left(3^{*}\right)
$$

Niiniluoto (1973), however, demonstrates in detail that (a) and (b) cannot be jointly accepted, and hence that $\left(1^{*}\right) \Rightarrow\left(3^{*}\right)$ does not hold, no matter how "testable" in $\left(2^{*}\right)$ is explicated. Note in particular that $\left(1^{*}\right) \Rightarrow\left(3^{*}\right)$ amounts to the claim that if a theory achieves inductive systematization, then it also achieves deductive systematization. But that is certainly a long way from being unproblematic.

In sum, the Hempel-Scheffler argument can, after all, be defended against the critiques, improved, and shown to be basically correct. As such, it arguably presents an unsurmountable problem for the claim that the cognitive content of a theory can be captured by its Ramsey sentence.

\section{Underdetermination Revisited}

The above argument provides a bonus: at the outset of this paper, the underdetermination challenge was mentioned, as well as the standard response that by also taking into account inductive and not only deductive relations between theories and observation, at least the massive and radical type of underdetermination can be ruled out. Although the suggestion appears plausible, it has apparently never been rigorously demonstrated that it would make a difference. However, our above considerations illuminate this idea, too: our two theories $S_{1}$ and $S_{2}$ are empirically equivalent when it comes to deductive relations (namely, neither of them deductively entails any observational sentences) - the choice between them is therefore deductively underdetermined. Observation can nevertheless speak in favour of one of them at the expense of the other. For example, observing $O_{1}(a)$ and $O_{2}(a), O_{1}(b)$ and $O_{2}(b)$, etc. would provide an inductive confirmation of $S_{1}$ and disconfirmation of $S_{2}$. Our argument thus also provides a clear example of a pair of theories such that the choice between them is deductively but not inductively underdetermined. 


\section{Acknowledgements}

Earlier versions of this paper have been presented in the philosophy of science seminars in Helsinki and Athens. I would like to thank both audiences for the useful feedback. I am especially indebted to Stathis Psillos for his valuable comments. Finally, I am grateful to the two anonymous reviewers for their helpful comments.

\section{References}

Ainsworth, P.: 2009. 'Newman's Objection', The British Journal for the Philosophy of Science 60, 135-171.

Bohnert, H.G.: 1968. 'In Defense of Ramsey's Elimination Method', Journal of Philosophy 65, 275281.

Braddon-Mitchell, D. and Nola R. (eds.): 2009. Conceptual Analysis and Philosophical Naturalism, MIT Press, Cambridge MA.

Cruse, P.: 2005. 'Ramsey Sentences, Structural Realism and Trivial Realization', Studies in History and Philosophy of Science 36,557-576.

Demopoulos, W. and Friedman, M.: 1985. 'Critical notice: Bertrand Russell's The Analysis of Matter: Its Historical Context and Contemporary Interest', Philosophy of Science 52, 621-639.

Hempel, C.G.: 1945. 'Studies in the Logic of Confirmation', Mind 54, 1-26, 97-121. Reprinted in Hempel 1965.

Hempel, C.G.: 1958. 'The Theoretician's Dilemma: A Study in the Logic of Theory Construction', Reprinted in Hempel 1965.

Hempel, C.G.: 1965. Aspects of Scientific Explanation and Other Essays in the Philosophy of Science. The Free Press, New York.

Hesse, M.: 1970a. 'Theories and the Transitivity of Confirmation', Philosophy of Science 37, 50-63.

Hesse, M.: 1970b. 'An Inductive Logic of Theories', Minnesota Studies in the Philosophy of Science IV, University of Minnesota Press, Minneapolis, 164-180.

Hooker, C.A.: 1968. 'Craigian Transcriptionism', American Philosophical Quarterly 5, 152-163.

Ketland, J.: 2004. 'Empirical Adequacy and Ramsification', The British Journal for the Philosophy of Science 55: 409-424.

Ketland, J.: 2009. 'Empirical Adequacy and Ramsification, II'. In A. Heike \& H Leitgeb (eds.) Reduction - Abstraction - Analysis. Proceedings of 31 st International Wittgenstein Symposium, ontos verlag, Frankfurt, 29-45.

Ladyman, J.: 2002. Understanding Philosophy of Science, Routledge, London.

Ladyman, J.: 2008. 'Structural Realism', in Ed Zalta (ed.) Stanford Encyclopaedia of Philosophy. http://plato.stanford.edu/entries/structural-realism/

Laudan, L.: 1981. 'A Confutation of Convergent Realism', Philosophy of Science 48, 19-49.

Laudan, L.: 1990. 'Demystifying Underdetermination', Minnesota Studies in the Philosophy of Science 14, 267-297

Laudan, L.: 1998. 'Underdetermination', in E. Craig (ed.) The Routledge Encyclopedia of Philosophy, Routledge, London.

Melia, J. and Saatsi, J.: 2006. 'Ramsification and Theoretical Content', The British Journal for the Philosophy of Science 57, 561-585.

Newman, M. H. A.: 1928. "Mr. Russell's Causal Theory of Perception", Mind 37, 137-48.

Niiniluoto, I. (1972). "Inductive Systematization: Definition and a Critical Survey", Synthese 25, 2581. 
Niiniluoto, I. (1973). "Empirically Trivial Theories and Inductive Systematization", in R. Bogdan and I. Niiniluoto (eds.) Logic, Language and Probability. (A selection of papers from the IVth International Congress in Logic, Methodology and the Philosophy of Science.) Reidel, 108-114. Psillos, S. (2005). "Underdetermination”, Encyclopedia of Philosophy, 2nd Edition, Gale MacMillan Reference.

Psillos, S. (2006). "Ramsey's Ramsey Sentences". In M.C. Galavotti ed., Cambridge and Vienna: Frank P. Ramsey and the Vienna Circle, pp. 67-90. Dordrecht: Springer.

Ramsey, F. (1931). "Theories", in F. Ramsey, The Foundations of Mathematics and Other Essays. (Edited by R. B. Braithwaite). London: Routledge and Kegan Paul 1931, 212-236.

Scheffler, I. (1963). The Anatomy of Inquiry, Alfred A. Knopf, New York.

Scheffler, I. (1968). "Reflections on the Ramsey Method", Journal of Philosophy 65, 269-74.

Skyrms, B. (1966). "Nomological Necessity and the Paradoxes of Confirmation", Philosophy of Science 33, 230-49.

Stegmüller, W. 1970. Theorie und Erfarung. Probleme und Resultate der Wissenschaftstheorie und analytischen Philosophie, Vol. II. Springer-Verlag, Berlin.

Tuomela, Raimo (1973). Theoretical Concepts. Springer.

Tuomela, Raimo (1974). "Review", Journal of Symbolic Logic 39, 617-619.

Worrall, J. (1989). "Structural Realism: The best of Both Worlds?" Dialectica 43, 99-124. Reprinted in D. Papineau, ed., The Philosophy of Science, pp. 139-165. Oxford: Oxford University Press.

Worrall, J. and E. Zahar (2001). "Ramseyfication and Structural Realism", Appendix IV, in Zahar (2001), pp. 236-251.

Zahar, E. (2001). Poincaré's Philosophy: From Conventionalism to Phenomenology. Chicago and La Salle: Open Court.

\section{Notes}

1 Obviously, Newman himself did not address Ramsey sentences, as his paper predates Ramsey's seminal paper (Ramsey 1931). Nevertheless, the contemporary objection to Ramsification in question owes so much to Newman's argument that it is appropriate to call it "the Newman objection"; cf. (Demopoulos \& Friedman 1985).

${ }^{2}$ How exactly "empirical adequacy" should be explicated here turns out to be more complicated than initially assumed; see (Ketland 2004, 2009).

3 This theory can be viewed as a simplified version of Hempel's theory of 'white phosphorus' (Hempel 1958, 214-15).

${ }^{4}$ Strictly speaking, of course, inductive systematization between statements, say, $O_{1}(a)$ and $O_{2}(a)$, has not been defined. Inductive systematization is rather defined with respect to the language $L_{O}$ which includes them. We take $O_{1}(a)$ as $e$, put either $O_{1}(a)$ or $\neg O_{2}(a)$ for $h$, and finally, take either $S_{1}$ or $S_{2}$ or the Ramsey sentence of one or the other as $S$, in the definition of inductive systemazation, 
and ask whether $h$ is inducible from $(S \wedge e)$ or not. If it is, then we have achieved inductive systemazation with respect to $L_{O}$.

${ }^{5}$ Put $h \leftrightarrow(e \wedge b)$. Then $h$ is inducible from from $e$ by Converse Entailment, and $b$ is deducible from $h$. Hence $e$ confirms $b$, by Special Consequence. But $e$ and $b$ could be just any given statements; therefore a relation of confirmation satisfying both these conditions is trivial.

${ }^{6}$ This problem was identified, not by the opponents of Scheffler, but by Niiniluoto (1972) - just in order to reply to it. 